archives-ouvertes

\title{
An expression of the magnetic co-energy adapted to magnetostatic volume integral formulations - application to the magnetic force computation
}

Quentin Debray, Gérard Meunier, Olivier Chadebec, Jean-Louis Coulomb, Anthony Carpentier

\section{To cite this version:}

Quentin Debray, Gérard Meunier, Olivier Chadebec, Jean-Louis Coulomb, Anthony Carpentier. An expression of the magnetic co-energy adapted to magnetostatic volume integral formulations - application to the magnetic force computation. International Journal of Applied Electromagnetics and Mechanics, 2019, 59 (1), pp.3-8. 10.3233/JAE-171110 . hal-02350920

\section{HAL Id: hal-02350920 \\ https://hal.archives-ouvertes.fr/hal-02350920}

Submitted on 14 Nov 2019

HAL is a multi-disciplinary open access archive for the deposit and dissemination of scientific research documents, whether they are published or not. The documents may come from teaching and research institutions in France or abroad, or from public or private research centers.
L'archive ouverte pluridisciplinaire HAL, est destinée au dépôt et à la diffusion de documents scientifiques de niveau recherche, publiés ou non, émanant des établissements d'enseignement et de recherche français ou étrangers, des laboratoires publics ou privés. 


\title{
An expression of the Magnetic Co-Energy adapted to MagnetoStatic Volume Integral Formulations - Application to the Magnetic Force computation
}

\author{
Quentin Debray ${ }^{\mathrm{a}, \mathrm{b}, *}$, Gerard Meunier ${ }^{\mathrm{a}}$, Olivier Chadebec ${ }^{\mathrm{a}}$, Jean-Louis Coulomb ${ }^{\mathrm{a}}$ and \\ Anthony Carpentier ${ }^{b}$ \\ ${ }^{\text {a } U n i v . ~ G r e n o b l e ~ A l p e s, ~ C N R S, ~ G r e n o b l e ~ I N P *, ~ G 2 E l a b, ~ F-38000 ~ G r e n o b l e, ~ F r a n c e ~}$ \\ ${ }^{\mathrm{b}}$ Altair Engineering, 15 chemin de Malacher, 38340 Meylan, France
}

\begin{abstract}
This paper provides a new expression of the magnetic co-energy for ferromagnetic materials. This expression is very well suited to integral methods but can as well be used as a post function for the Finite Elements Method (FEM). First the context of this work and the expression of the co-energy will be introduced. Second, two examples will be treated with the proposed method and the performances will be compared with the FEM.
\end{abstract}

Keywords: Volume Integral Method, Magnetic Co-Energy, Force Computation, Torque Computation

\section{Introduction}

For a few years, Volume Integral Method (VIM) has been widely studied [4][1][2][3] and is know today as a good alternative to the Finite Elements Method (FEM) for solving magnetostatic problems in the presence of non-linear materials. While the FEM needs the geometry to be completely meshed, the VIM only requires the meshing of active materials. Moreover, while the FEM only takes into acount local approximated interactions between Degrees Of Freedom (DOF), VIM considers Green kernel type interaction. Those types of interactions allow to be as efficient as possible on a given mesh since the quality of the calculation of the interaction is very high : indeed, the interaction not only the short distance interactions are taken into account, but also the long distances interractions thanks to the Green kernel type interaction. Nevertheless, since the unknows of the VIM are inside the active materials, this methods yields the magnetic field (or the magnetic induction) only in the active materials. The information regarding the magnetic field in the air around the active materials can be obtained by calculation but has a cost. Thus the classical methods of force computation (Maxwell Stress Tensor [7], Virtual Works method [5]) are not usable (or at least not as efficient as with the FEM) and we must find specialy adapted techniques to compute the forces. First we will provide and demonstrate an expression of the magnetic

\footnotetext{
"Corresponding author: Quentin Debray, Altair Engineering, 15 chemin de Malacher, 38340 Meylan, France, E-mail: qdebray@altair.com
} 
co-energy (which can be used to compute the magnetic force [6]) that requires only the information of the magnetic field inside the active materials. Second we will validate this expression by calculating the force on the mobile part of a contactor.

\section{Formula}

The magnetic co-energy is defined via the relation :

$$
W_{m a g}^{c o}=\int_{\Omega}\left(\int_{0}^{\mathbf{H}} \mathbf{B}\left(\mathbf{H}^{\prime}\right) \cdot \mathrm{d} \mathbf{H}^{\prime}\right) \mathrm{d} \Omega
$$

where $\Omega$ is the whole study domain (which includes the air, and the ferro-magnetic materials). Our goal is to bring this expression back to the domain $\Omega_{m}$ which is the domain on which the VIM yields the magnetic field :

$$
\Omega_{m}=\Omega_{p m}+\Omega_{\text {ferro }}
$$

where $\Omega_{p m}$ is the domain containing permanents magnets and $\Omega_{\text {ferro }}$ is the ferromagnetic materials. An illustration of those domains can be found figure (1a) and (1b).

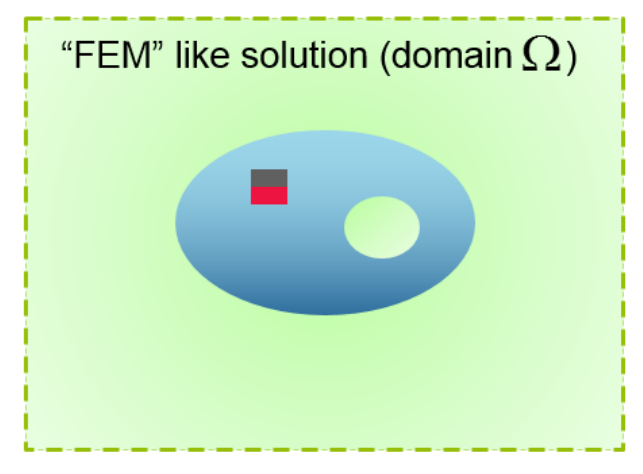

(a) FEM

\section{"VIM" like solution (domain $\Omega_{m}$ )}

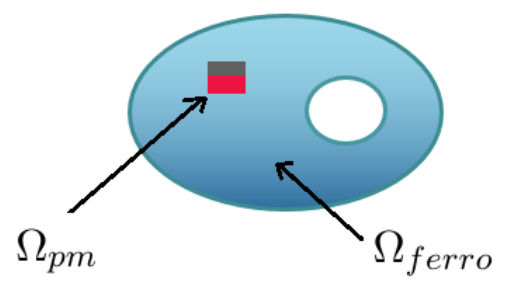

(b) VIM

Fig. 1. Description of the two types of domains (FEM and VIM)

\section{Linear materials}

We have :

$$
\mathbf{B}=\mu \mathbf{H}+\mathbf{B}_{r}
$$

which yields, for the expression of the co-energy :

$$
W_{m a g}^{c o}=\int_{\Omega} \frac{1}{2} \mu \mathbf{H}^{2}+\mathbf{B}_{r} \cdot \mathbf{H} \mathrm{d} \Omega
$$

that can be decomposed in : 


$$
W_{m a g}^{c o}=\int_{\Omega} \frac{1}{2} \mu \mathbf{H}^{2} \mathrm{~d} \Omega+\int_{\Omega_{p m}} \mathbf{B}_{r} \cdot \mathbf{H} \mathrm{d} \Omega_{p m}
$$

since $\mathbf{B}_{r}=0$ outside of the permanent magnets. The equation 3 allows to modify the first term of equation 5 :

$$
W_{m a g}^{c o}=\int_{\Omega} \frac{1}{2}\left(\mathbf{B H}-\mathbf{B}_{r} \mathbf{H}\right) \mathrm{d} \Omega+\int_{\Omega_{p m}} \mathbf{B}_{r} \cdot \mathbf{H} \mathrm{d} \Omega_{p m}
$$

which leads to :

$$
W_{m a g}^{c o}=\int_{\Omega} \frac{1}{2} \mathbf{B H} \mathrm{d} \Omega+\int_{\Omega_{p m}} \frac{1}{2} \mathbf{B}_{r} \mathbf{H} \mathrm{d} \Omega_{p m}
$$

Using the equations $\mathbf{H}=\mathbf{H}_{0}-\nabla \varphi$ and $\mathbf{B}=\nabla \times \mathbf{A}=\nabla \times\left(\mathbf{A}_{0}+\mathbf{A}_{m}\right)$ where $\varphi$ is the scalair potential, $\mathbf{A}_{0}$ is the vector potential induced by the sources and $\mathbf{A}_{m}$ is the vector potential induced by the magnetization of the material $\Omega_{m}$, one can write :

$$
\int_{\Omega} \frac{1}{2} \mathbf{B H} \mathrm{d} \Omega=\int_{\Omega} \frac{1}{2} \mathbf{B} \mathbf{H}_{0}-\int_{\Omega} \mathbf{B} \nabla \varphi \mathrm{d} \Omega
$$

We moreover use that :

$$
\int_{\Omega} \mathbf{B} \nabla \varphi \mathrm{d} \Omega=0
$$

Indeed the divergence theorem allows to re-write the previous equation as :

$$
\int_{\Omega} \mathbf{B} \nabla \varphi \mathrm{d} \Omega=\int_{\Gamma} \varphi B_{n} \mathrm{~d} \Gamma+\int_{\Omega} \varphi \nabla \cdot \mathbf{B} \mathrm{d} \Omega
$$

and considering the facts that $B_{n}$ and $\varphi$ are continuous at the interfaces, that $\nabla \cdot \mathbf{B}=0$ and $\varphi=0$ to the infinite, we can conclude that :

$$
\int_{\Omega} \frac{1}{2} \mathbf{B H} \mathrm{d} \Omega=\int_{\Omega} \frac{1}{2} \mathbf{B} \mathbf{H}_{0} \mathrm{~d} \Omega
$$

Thus, by using that $\mathbf{B}=\nabla \times\left(\mathbf{A}+\mathbf{A}_{m}\right)$, we can write :

$$
\int_{\Omega} \frac{1}{2} \mathbf{B H}_{0} \mathrm{~d} \Omega=W_{0}+\int_{\Omega} \frac{1}{2} \nabla \times \mathbf{A}_{m} \mathbf{H}_{0} \mathrm{~d} \Omega
$$

where $W_{0}$ is the magnetic energy which would be generated by the coils in absence of any magnetic materials :

$$
W_{0}=\frac{1}{2} \int_{\Omega} \nabla \times \mathbf{A}_{0} \mathbf{H}_{0}
$$


This energy can be computed via :

$$
W_{0}=\sum_{k=1}^{N_{c}} \sum_{l=1}^{N_{c}} \frac{1}{2} L_{k l} I_{l} I_{k} \quad \text { with } \quad L_{k l}=\frac{\mu_{0}}{2 \pi} \int_{\Omega_{k}^{C}} j_{0}^{k} \int_{\Omega_{l}^{C}} j_{0}^{l} G(\mathbf{r}) \mathrm{d} \Omega_{l}^{C} \mathrm{~d} \Omega_{k}^{C}
$$

where $L_{k l}$ is the mutual inductance between the coil $l$ and $k$ without active materials, $N_{c}$ is the umber of coil and $I_{k}$ is the current going through coil $k . G(\mathbf{r})$ is the expression of the Green kernel :

$$
\begin{array}{r}
G(\mathbf{r})=\log \left(\frac{1}{\|\mathbf{r}\|}\right) \text { in } 2 \mathrm{D} \\
G(\mathbf{r})=-\frac{\mathbf{r}}{\|\mathbf{r}\|^{2}} \text { in } 3 \mathrm{D}
\end{array}
$$

$\Omega_{k}^{C}$ is the domain of the coil $k$, and $j_{0}^{k}$ is the current density in the coil $k$ inducing a $1 \mathrm{~A}$ current. The double integral shown equation (14 right) can be computed with a semi-analytical approach : the first integral on the region $\Omega_{l}^{C}$ can be calculated analyticaly [8] while the second integral is calculated with an adaptative Gauss point integration approach. The equation (12) can then be modified using relation $\mathbf{B}=\nabla \times \mathbf{A}_{m}+\nabla \times \mathbf{A}_{0}=\mu_{0}(\mathbf{H}+\mathbf{M})=\mu_{0}\left(\mathbf{H}_{0}-\nabla \varphi+\mathbf{M}\right):$

$$
\int_{\Omega} \frac{1}{2} \nabla \times \mathbf{A}_{m} \mathbf{H}_{0} \mathrm{~d} \Omega=\int_{\Omega} \mathbf{H}_{0} \cdot \mu_{0}(-\nabla \varphi+\mathbf{M}) \mathrm{d} \Omega
$$

We use once again the fact that $\varphi=0$ to the infinite and $\nabla \cdot\left(\mu_{0} \mathbf{H}_{0}\right)=0$ to prove that :

$$
\int_{\Omega} \mathbf{H}_{0} \cdot \mu_{0} \nabla \varphi \mathrm{d} \Omega=0
$$

and conclude that :

$$
\int_{\Omega} \frac{1}{2} \nabla \times \mathbf{A}_{m} \mathbf{H}_{0} \mathrm{~d} \Omega=\int_{\Omega} \mathbf{H}_{0} \cdot \mu_{0} \mathbf{M} \mathrm{d} \Omega=\int_{\Omega_{m}} \mathbf{H}_{0} \cdot \mu_{0} \mathbf{M} \mathrm{d} \Omega_{m}
$$

Since $\mathbf{M}=0$ outside of the active materials. We finally get the expression of the magnetic co-energy in linear materials which can be computed by integrating over $\Omega_{m}$ only :

$$
W_{m a g}^{c o}=W_{0}+\int_{\Omega_{m}} \frac{1}{2} \mu_{0} \mathbf{H}_{0} \mathbf{M} \mathrm{d} \Omega_{m}+\int_{\Omega_{p m}} \frac{1}{2} \mathbf{B}_{r} \mathbf{H} \mathrm{d} \Omega_{p m}
$$

\section{General case}

We start once again from the co-energy definition :

$$
W_{\text {mag }}^{c o}=\int_{\Omega}\left(\int_{0}^{\mathbf{H}} \mathbf{B}\left(\mathbf{H}^{\prime}\right) \cdot \mathrm{d} \mathbf{H}^{\prime}\right) \mathrm{d} \Omega
$$

and we separate this expression in two domains : 
- $\Omega_{\text {air }}$ which corresponds to the air in the domain $\Omega$

- $\Omega_{m}$ which has already been defined

We thus have :

$$
W_{\text {mag }}^{c o}=\int_{\Omega_{m}}\left(\int_{0}^{\mathbf{H}} \mathbf{B}\left(\mathbf{H}^{\prime}\right) \cdot \mathrm{d} \mathbf{H}^{\prime}\right) \mathrm{d} \Omega_{m}+\int_{\Omega_{a i r}}\left(\int_{0}^{\mathbf{H}} \mathbf{B}\left(\mathbf{H}^{\prime}\right) \cdot \mathrm{d} \mathbf{H}^{\prime}\right) \mathrm{d} \Omega_{a i r}
$$

to which we add and remove $\int_{\Omega} \frac{1}{2} \mathbf{B H} \mathrm{d} \Omega$ :

$$
\begin{aligned}
W_{m a g}^{c o}= & \int_{\Omega} \frac{1}{2} \mathbf{B H} \mathrm{d} \Omega+\int_{\Omega_{m}}\left(\int_{0}^{\mathbf{H}} \mathbf{B}\left(\mathbf{H}^{\prime}\right) \cdot \mathrm{d} \mathbf{H}^{\prime}\right) \mathrm{d} \Omega_{m}-\int_{\Omega_{m}} \frac{1}{2} \mathbf{B} \mathbf{H} \mathrm{d} \Omega_{m}+ \\
& \int_{\Omega_{a i r}}\left(\int_{0}^{\mathbf{H}} \mathbf{B}\left(\mathbf{H}^{\prime}\right) \cdot \mathrm{d} \mathbf{H}^{\prime}\right) \mathrm{d} \Omega_{a i r}-\int_{\Omega_{a i r}} \frac{1}{2} \mathbf{B} \mathbf{H} \mathrm{d} \Omega_{a i r}
\end{aligned}
$$

The two terms regarding $\Omega_{\text {air }}$ cancel each other and we can use the developments seen in the linear materials paragraph for the term $\int_{\Omega} \frac{1}{2} \mathbf{B H} \mathrm{d} \Omega$ to finaly get :

$$
W_{m a g}^{c o}=W_{0}+\int_{\Omega_{m}}\left[\frac{1}{2} \mu_{0} \mathbf{H}_{0} \cdot \mathbf{M}-\frac{1}{2} \mathbf{B} \cdot \mathbf{H}+\int_{0}^{\mathbf{H}} \mathbf{B}\left(\mathbf{H}^{\prime}\right) \mathrm{d} \mathbf{H}^{\prime}\right] \mathrm{d} \Omega_{m}
$$

The force can be computed from the magnetic co-energy along the direction of displacement as :

$$
F_{s}=\frac{\mathrm{d} W_{m a g}^{c o}}{\mathrm{~d} s}
$$

where $s$ is the displacement of the mobile on which the force is calculated. The current flowing through the eventual coils of the problem has to remain constant.

\section{Validation}

The test case is the 2D contactor displayed figure (2a). All the dimensions given in the figure (2a) are all in $\mathrm{mm}$. The mobile part is shifted from $-5 \mathrm{~mm}$ to $5 \mathrm{~mm}$ in the horizontal direction. The force according this direction is computed for 100 position. The profile of the magnetic co-energy and the force along the (Ox) axis yielded by the proposed method are available figure (2b). We decided to compare the efficiency of the proposed method (VIM for the field computation and derivation of the magnetic co-energy for the force computation) to the FEM coupled to the virtual works method. We used a converged FEM solution as a reference and we calculated the mean and maximum error for the two methods. The results are availlable figure (3a). The computation times are displayed as well figure (3b).

The results of this study shows that with the same amount of unknows in the active materials (abscissa of the graph found figure (3a)) the combination of the VIM with the derivation of the magnetic co-energy (DCE) yields results as good as the FEM coupled with the virtual works (VW) method. Nevertheless, when the number of unknows is low (less than 1000 unknows in our case), the coupling VIM+DCE is 
Ferro-magnetic material $\mu_{r}=1000, J_{s}=1 T$

Linear magnet $\mu_{r}=1, B_{r}=1 T$

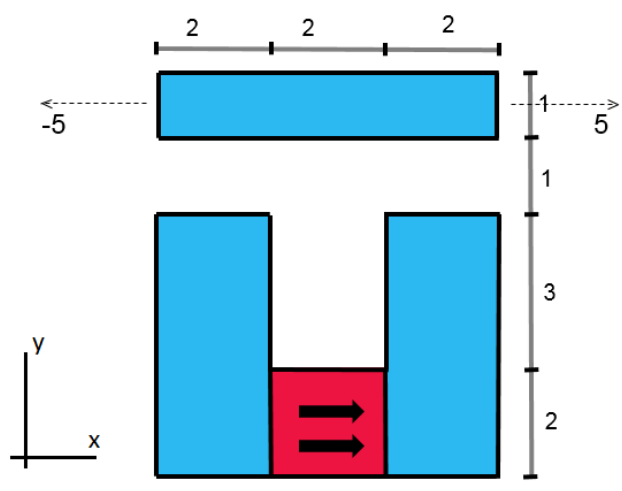

(a) Test case

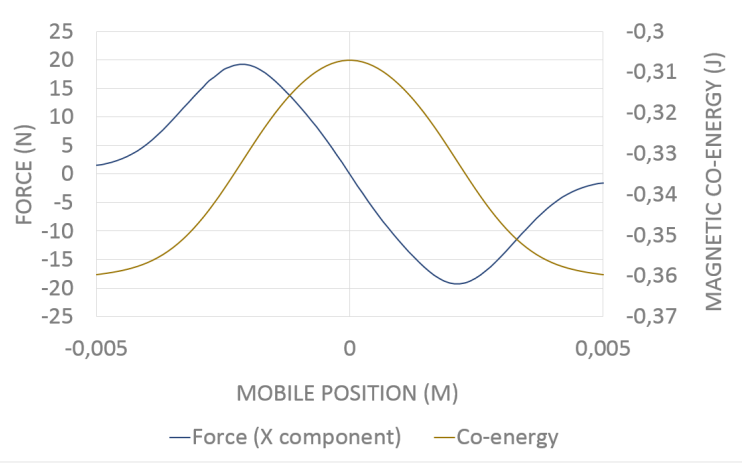

(b) Magneti co-energy and force profile

Fig. 2. Description of the test case

far more effective than the FEM+VW coupling. Indeed, computing the integral (24) is extremely cheap and the VIM is very effective for problems with few unknowns. Moreover, one can notice that in a case with moving parts, the VIM has the advantage of not requirering remeshing the domain, contrairy to the FEM. If the number of unknows if high, the VIM is not competitive anymore since the complexity is $O\left(n^{2}\right)$ while the complexity of the FEM is $O(n)$.

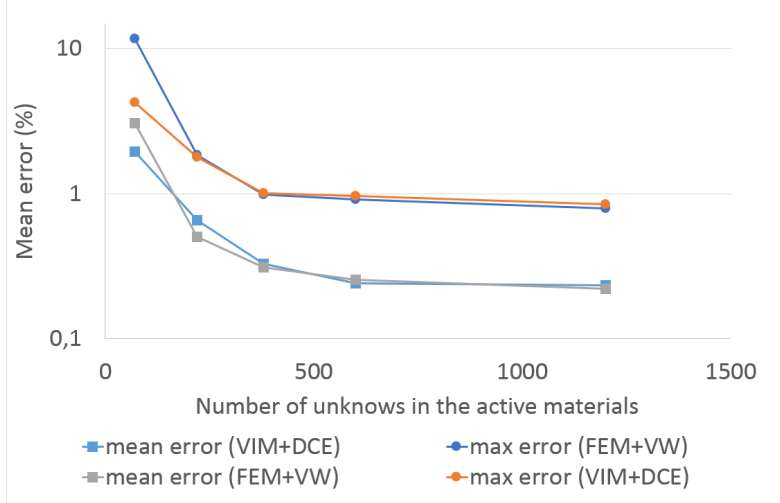

(a) Mean and maximal error

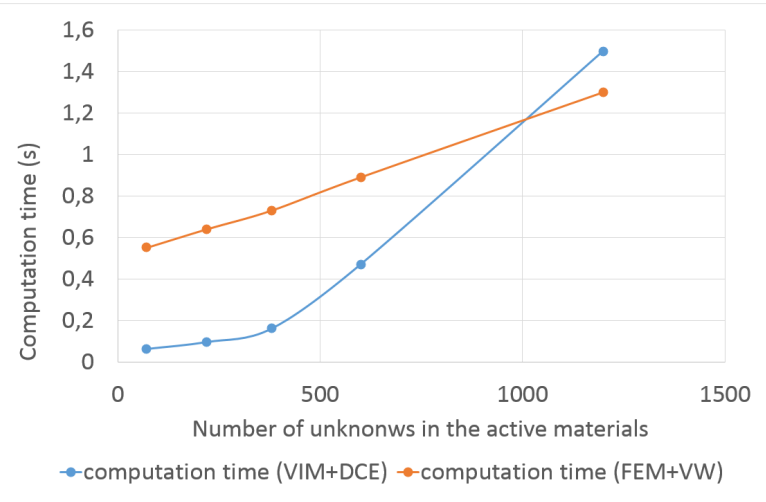

(b) Computation time

Fig. 3. Description of the test case

\section{Conclusion}

An original expression of the magnetic co-energy has been presented. This expression is adapted to the volume integral method since it only requires information in the active materials. This expression can be used to compute efficiently the force or the torque applied to any magneto-static device. The coupling of 
this method with the VIM shows great promises, especially in a context of pre-design where fasts solving methods are required, yielding fairly accurate results. One can moreover notice that this expression of the magnetic co-energy can be used in coupling with the finite elements method as well, the information in the air is simply ignored. No experiments have been done in this area yet.

\section{References}

[1] V. Le-Van, G. Meunier, O. Chadebec and J. M. Guichon, "A Volume Integral Formulation Based on Facet Elements for Nonlinear Magnetostatic Problems," in IEEE Transactions on Magnetics, vol. 51, no. 7, pp. 1-6, July 2015

[2] A. Carpentier, O. Chadebec, N. Galopin, G. Meunier and B. Bannwarth, "Resolution of Nonlinear Magnetostatic Problems With a Volume Integral Method Using the Magnetic Scalar Potential," in IEEE Transactions on Magnetics, vol. 49, no. 5, pp. 1685-1688, May 2013

[3] V. Le-Van, G. Meunier, O. Chadebec and J. M. Guichon, "A Magnetic Vector Potential Volume Integral Formulation for Nonlinear Magnetostatic Problems," in IEEE Transactions on Magnetics, vol. 52, no. 3, pp. 1-4, March 2016

[4] G. Meunier, O. Chadebec and J. M. Guichon, "A Magnetic Flux-electric Current Volume Integral Formulation Based on Facet Elements for Solving Electromagnetic Problems," in IEEE Transactions on Magnetics, vol. 51, no. 3, pp. 1-4, March 2015

[5] J. Coulomb and G. Meunier, Finite element implementation of virtual work principle for magnetic or electric force and torque computation, IEEE Transactions on Magnetics, vol?20, no. 5, pp. 1894-1896, sept 1984

[6] W. N. Fu, P. Zhou, D. Lin, S. Stanton and Z. J. Cendes, "Magnetic force computation in permanent magnets using a local energy coordinate derivative method," in IEEE Transactions on Magnetics, vol. 40, no. 2, pp. 683-686, March 2004.

[7] A. Bermudez, A. L. Rodriguez and I. Villar, "Extended Formulas to Compute Resultant and Contact Electromagnetic Force and Torque From Maxwell Stress Tensors," in IEEE Transactions on Magnetics, vol. 53, no. 4, pp. 1-9, April 2017.

[8] E. Durand, Magnetostatique, 1968 